\title{
Infrared Vibrational Nanospectroscopy by Self-Referenced Interferometry
}

\author{
Supporting Information
}

\begin{abstract}
Benjamin Pollard, ${ }^{\dagger}$ Francisco C. B. Maia, ${ }^{\ddagger}$ Markus B. Raschke, ${ }^{* \dagger}$ and Raul O. Freitas*,‡

Department of Physics, Department of Chemistry, and JILA, University of Colorado, Boulder, Colorado 80309, USA, and Brazilian Synchrotron Light Laboratory, Campinas, SP, Brazil
\end{abstract}

E-mail: markus.raschke@colorado.edu; raul.freitas@lnls.br

\section{SINS experiment}

Self-heterodyne SINS experiments were performed in the IR1 beamline of the LNLS ${ }^{1}$, a 1.37 $\mathrm{GeV}$ second-generation synchrotron storage ring that operates in decay mode with $250 \mathrm{~mA}$ maximum current. A set of five gold-coated mirrors compose the beamline primary optics. IR broadband radiation with a divergence of $80 \mathrm{mrad}$ by $30 \mathrm{mrad}$ (horizontal $\times$ vertical) from the $1.67 \mathrm{~T}$ bending magnet is spatially extracted by a non-cooled flat mirror with a central slot. The IR beam is then focused at 7 meters from the source by a pair of mirrors (conical and cylindrical). Coma aberration from the source depth (dipole extraction length) is corrected by the conical mirror. Around $1.5 \mathrm{~m}$ downstream from the focal point, a pair of

\footnotetext{
* To whom correspondence should be addressed

${ }^{\dagger} \mathrm{CU}$-Boulder

${ }^{\ddagger} \mathrm{LNLS}$

${ }^{1}$ www.lnls.cnpem.br/beamlines/ir1/
} 
cylindrical mirrors colimate the beam and deliver it to the optical table. After the primary optics, the collimated beam is $29 \mathrm{~mm}$ by $9 \mathrm{~mm}$ with a divergence of $0.5 \mathrm{mrad}$ by $1.0 \mathrm{mrad}$ (horizontal $\times$ vertical, respectively). The beam is apertured to $12 \mathrm{~mm}$ by $9 \mathrm{~mm}$ as it enters the $s$-SNOM microscope (NeaSNOM, Neaspec GmbH) that was slightly modified in order to incorporate a symmetric Michelson interferometer earlier in the beam path. The beam is first incident on an uncoated ZnSe beamsplitter (Fig. 1a) that divides the beam into the moveable arm (or reference arm) and static arm of the interferometer. In the reference arm, a piezo linear stage (P.629.1 PIHera, Physik Instrumente GmbH \& Co.) translates the reference mirror (moveable mirror in Fig. 1a) in order to produce up to $3000 \mu \mathrm{m}$ optical path difference $\left(3.3 \mathrm{~cm}^{-1}\right.$ maximum spectral resolution). The microscope is enclosed and purged with nitrogen to remove water and carbon dioxide and to increase thermal stability. The two beams from the interferometer are focused by a 0.45 NA parabolic mirror (tip objective) designed for the NeaSNOM microscope. Both beams from reference and fixed arms are focused onto the apex of an AFM tip (Nanosensors, PtSi-NCH). The alignment is done by monitoring the near-field signal (optical $2^{\text {nd }}$ harmonics) of each arm at a time (lower pannel of Fig. 1d). The AFM is operated in tapping-mode and the tip is set to oscillate at frequency $\Omega_{\text {tip }}$ which ranges from $250 \mathrm{kHz}$ to $350 \mathrm{kHz}$, inducing harmonics in the near-field scattered signal from the tip-sample interaction. ${ }^{1,2}$ Lock-in detection at higher harmonics of $\Omega_{\text {tip }}$ allows for discrimination of the near-field from far-field background ${ }^{3}$ For the detection, a mercury cadmium telluride (MCT) detector is positioned downstream for forward detection (Fig. 1a). The spectral range spans $700 \mathrm{~cm}^{-1}$ to $5000 \mathrm{~cm}^{-1}$, limited by the MCT detector response (MCT KLD-0.1, Kolmar Technologies Inc.) and the ZnSe beamsplitter.

The asymmetric setup mentioned in Fig. 1d uses the same light source, optical components, and instruments from the self-heterodyne SINS described above, except that the optical scheme that is based on an asymmetric Michelson interferometer as presented in Fig. S1. 


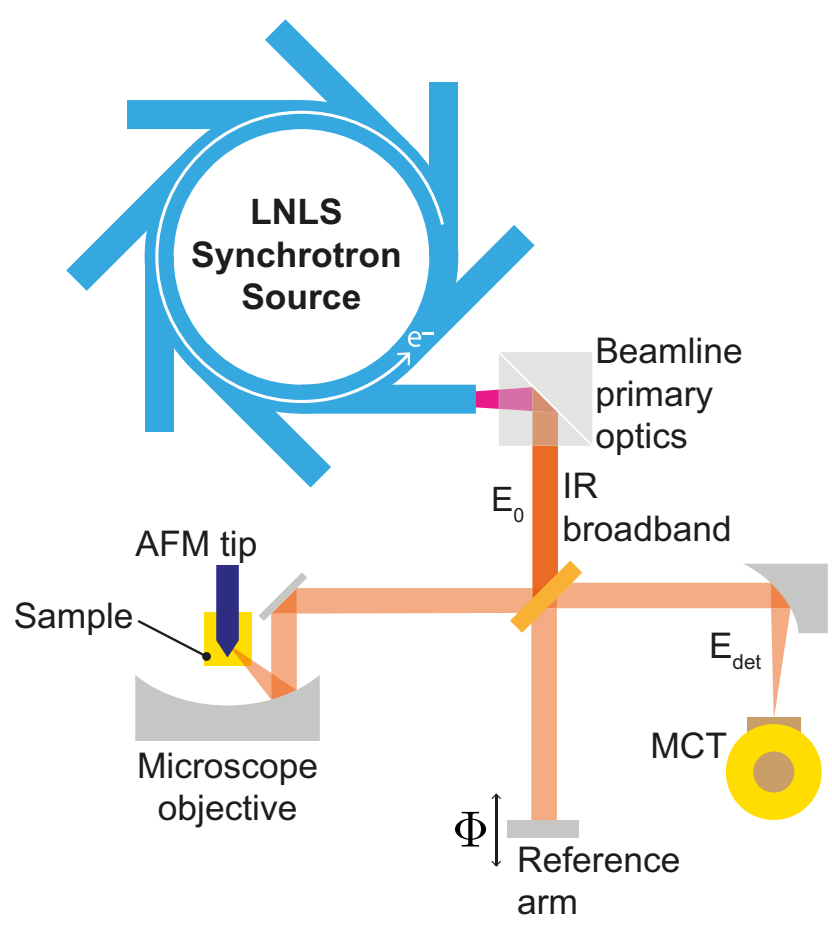

Figure S1: Asymmetric optical scheme for the SINS endstation in the IR1 beamline of LNLS. The broadband IR port from the synchrotron storage ring is used as light source for the $s$ SNOM experiment. Collimated beam from beamline primary optics enters an asymmetric Michelson interferometer in which the AFM is part of the static arm. The backscattered signal from tip-sample interaction experiences interferometric amplification when interfered with the far-field reference beam. Both beams from sample and reference arms are detected by the MCT. 


\section{Comparison to conventional $s$-SNOM}

The primary benefits of self-referenced interferometery when compared to traditional asymmetric configurations are a large increase in stability due to reduced sensitivity to fluctuations and drift, and an increased simplicity by simultaneously combining FTIR spectroscopy and microscopy as established in far-field techniques where the interferometer is completely decoupled from the microscope (here, the AFM). These improvements lead to increased sensitivity, shorter measurement times, and more robust data collection. To demonstrate, Fig. S2 shows broadband synchrotron data taken using both asymmetric and symmetric configurations on the same Au film on a Si substrate, plotting both the spectral signal-to-noise ratio (SNR) across a spectral range of interest and spectra themselves taken in sequence to illustrate stability. Eleven spectra (gray lines) on a Au surface were taken in sequence using each scheme, and then averaged (black/red lines). The SNR at each wavenumber was calculated by taking the ratio of the average signal to the standard deviation within the sequence. There is significantly less variation in the symmetric spectra than in the asymmetric ones, with an overall higher SNR for the symmetric dataset and more than twice as large for many wavenumbers. As noted in the main text, we see an overall $87 \%$ improvement in SNR over the asymmetric setup when integrated over the spectral range from $780 \mathrm{~cm}^{-1}$ to $1600 \mathrm{~cm}^{-1}$.

\section{Modelling self-referenced interferometry}

The near-field-scattered term $E_{\text {nf }}$ from the tip-sample interaction is directly proportional to the complex effective polarizability $\alpha$. There is also always a far-field scattered field $E_{\mathrm{ff}}$ arising from other far-field scatterers inside the confocal volume, that is, the region of space at the sample surface in which the beam can be considered "in-focus." Scatterers from both tip and sample contribute to this far-field scattering. We treat $E_{\mathrm{ff}}$ as directly proportional to $E_{0}$ with a complex constant factor $r$. The field $E_{\text {det }}$ reaching the detector (see Fig. 1a) 

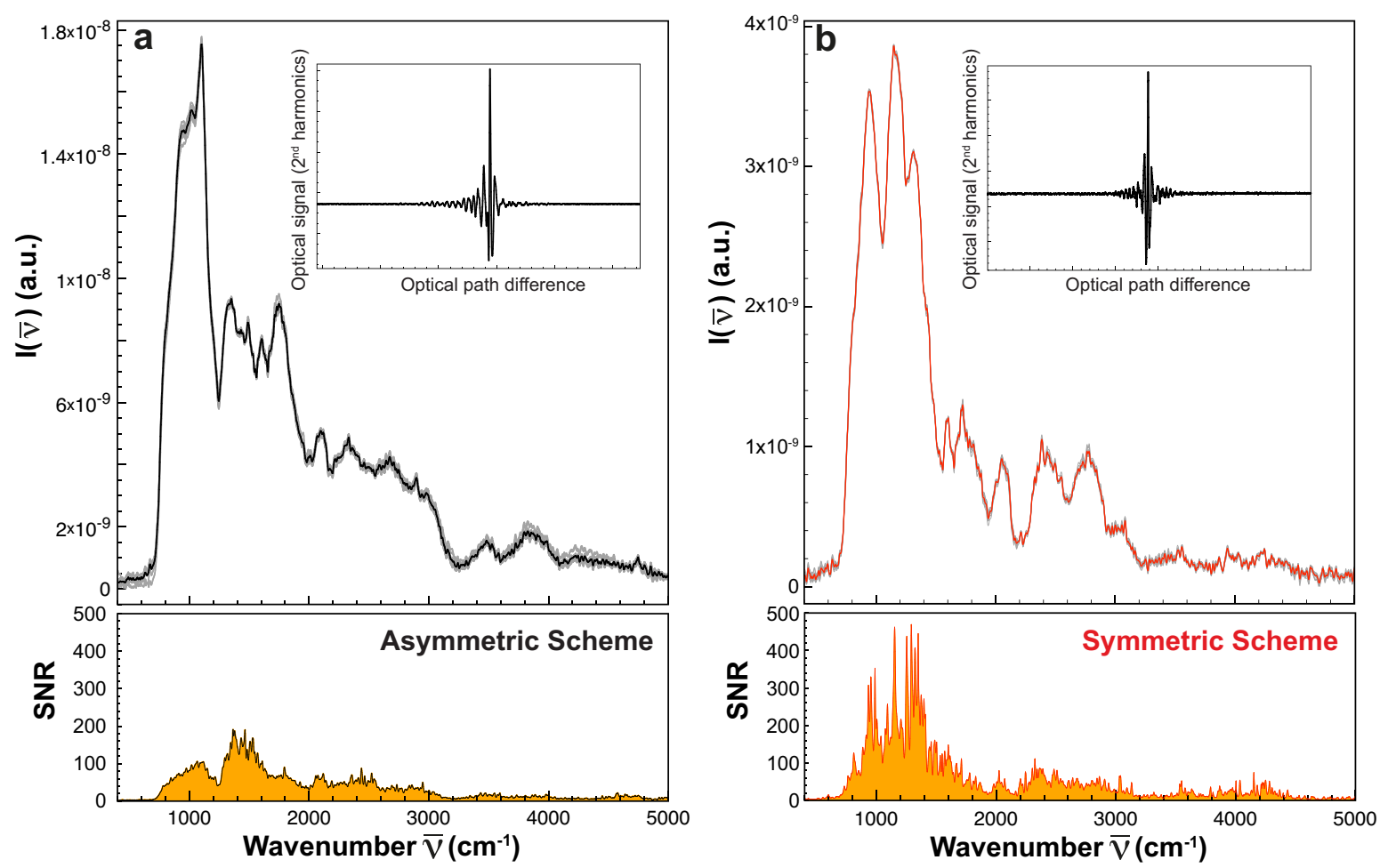

Figure S2: Spectra on Au taken with a traditional SINS setup (a) and self-heterodyne SINS (b). The spectra are the non-normalized output of a Fourier transform on interferograms such as those shown (inset). Measurements were repeated (gray) and averaged (black/red) to illustrate the improved stability of the symmetric configuration, which is further quantified in plots of SNR below each set of spectra. 
after tip-mediated near-field scattering is,

$$
E_{\mathrm{det}}=E_{\mathrm{nf}}+E_{\mathrm{ff}}=(\alpha+r) E_{0}
$$

with $\alpha$ described above and $r$ representing far-field scattering.

The resulting intensity $I$ measured by the detector follows to,

$$
I=\left|E_{\mathrm{det}}\right|^{2}=\left|E_{0}\right|^{2}\left(|r|^{2}+r^{*} \alpha+r \alpha^{*}+|\alpha|^{2}\right) .
$$

With $\alpha=A e^{i \phi}$, and $r=R e^{i \theta}$, in general,

$$
\begin{aligned}
I & =\left|E_{0}\right|^{2}\left(R^{2}+A^{2}+R A e^{i(\phi-\theta)}+R A e^{-i(\phi-\theta)}\right) \\
& =\left|E_{0}\right|^{2}\left(R^{2}+A^{2}+2 R A \cos (\phi-\theta)\right) .
\end{aligned}
$$

$R^{2}$ can be neglected as it is independent of the tip oscillation and therefore is eliminated via lock-in detection, and $A^{2}$ is negligibly small compared to the other terms. Thus,

$$
I(\bar{\nu})=2\left|E_{0}\right|^{2} R A(\bar{\nu}) \cos (\phi(\bar{\nu})-\theta)
$$

where we have explicitly highlighted the spectral dependence of the near-field scattering terms $A(\bar{\nu})$ and $\phi(\bar{\nu})$.

Similarly, considering a symmetric Michelson interferometer as shown in Fig. 1b, the field at point $\mathrm{P}$, corresponding to the driving $s$-SNOM field directly after the interferometer, is,

$$
E_{\mathrm{P}}=E_{\text {static }}+E_{\text {moving }}=\frac{1}{\sqrt{2}}\left(E_{0}+E_{0} e^{i \Phi}\right) .
$$

The resulting scattered field from replacing $E_{0} \rightarrow E_{\mathrm{P}}$ in Eq. S1 is given by,

$$
E_{\mathrm{det}}=\frac{1}{\sqrt{2}}\left(\alpha+\alpha e^{i \Phi}+r+r e^{i \Phi}\right) E_{0}
$$


The detector intensity $I$ then simplifies to,

$I=\left|E_{\mathrm{det}}\right|^{2}=\frac{1}{2}\left|E_{0}\right|^{2}\left(2+e^{i \Phi}+e^{-i \Phi}\right)\left(|r|^{2}+r^{*} \alpha+r \alpha^{*}+|\alpha|^{2}\right) \simeq 2\left|E_{0}\right|^{2} R A \cos (\phi-\theta)(1+\cos \Phi)$

A Fourier transform then yields,

$$
I(\bar{\nu})=2\left|E_{0}\right|^{2} R A(\bar{\nu}) \cos (\phi(\bar{\nu})-\theta)
$$

which is identical to Eq. S5.

\section{Combining SRIM with the Finite Dipole Model}

We extended the Self-Referenced Interferometery model (SRIM) described above to include a basic model of tip-sample interaction, the Finite Dipole Model (FDM). ${ }^{4}$ We again assume a vibrational resonant complex dielectric permittivity, $\epsilon=\widetilde{n}^{2}$, with a Lorentzian profile (Eq. S10), which now becomes an input of the FDM scattering. This dielectric permittivity enters into the quasi-static reflection coefficient $\beta$ (Eq. S11) which is inserted into the tip-sample effective polarizability $\alpha$ (Eq. S12):

$$
\begin{gathered}
\epsilon=\frac{\bar{\nu} \Gamma}{{\overline{\nu_{0}}}^{2}-\bar{\nu}^{2}-i \bar{\nu} \Gamma}, \\
\beta=\frac{\epsilon-1}{\epsilon+1},
\end{gathered}
$$

and

$$
\alpha=C\left(1+\frac{1}{2} \frac{f_{0} \beta}{1-f \beta}\right),
$$

The functions $f_{0}$ and $f$ of Eq. S12, which depend on parameters given by the tip-sample system, were evaluated in accordance with the typical values provided in the Ref. 4 . 
By inserting $\alpha$ into Eq. S9, we fit the PDMS amplitude of the $\mathrm{Si}-\mathrm{CH}_{3}$ vibrational resonance as shown in the Fig. $6 \mathrm{~b}$ of the main paper. Analogously to the fitting procedure of the basic model, which just adopted a raw Lorentzian for the scattering response, the extracted values from this combined model fit were used to create the extracted spectra which are plotted in Fig. 6d of the main text.

Combining SRIM with more sophisticated tip-sample interaction models is, conceptually, similarly straightforward. The resulting spectra from SRIM are the same complex values that are measured via traditional s-SNOM interferometery, and are the input values for a wide variety of established analysis and modeling techniques regarding tip-sample interactions, substrate effects, near-field scattering, etc. Thus one can use the resulting spectra from self-referenced measurements as the input for a wide variety of established s-SNOM models.

\section{Far-field experiments}

All far-field IR spectra (ATR-FTIR) were collected using the spectrometer Spectrum Two ${ }^{\mathrm{TM}}$ from Perkin Elmer (http://www.perkinelmer.com) in attenuated total reflectance geometry (ATR) using a diamond crystal. The reference spectra for background suppression were taken from a clean Au substrate (100 nm Au film sputtered on $\mathrm{Si}$ ) in the same ATR geometry.

Ellipsometry data on a thin-film of PMMA was taken using a Sentech Insturments GmbH spectral ellipsometer by Dr. Karsten Hinrichs, Department Berlin, Leibniz-Institut fur Analytische - ISAS - e.V., after Ref. ${ }^{5}$

\section{Fitting parameters}

For spectra with $\mathrm{N}$ adjacent resonances, we defined the Lorentzian resonator as a sum of separate Lorentzians which are weighted by a scaling factor $a_{j}$. All the Lorentzians share the same non resonant term $C$, with each representing a separate driven damped harmonic oscillator. 


$$
L(\bar{\nu})=\sum_{j=1}^{N} a_{j} L_{j}(\bar{\nu})=\sum_{j=1}^{N} a_{j}\left(\frac{\bar{\nu} \Gamma_{j}}{\bar{\nu}_{0, j}^{2}-\bar{\nu}^{2}-i \bar{\nu} \Gamma_{j}}+C\right)
$$

$L(\bar{\nu})$ is then used as a fitting function, as presented in the main text.

$$
I(\bar{\nu}) \simeq|L(\bar{\nu})| \cos (\operatorname{Arg}[L(\bar{\nu})]-\theta)
$$

Eq.S14 was used for all the fits of the paper, with fitting parameters presented in Table S1.

Table S1: Fit parameters.

\begin{tabular}{cccccc}
\hline Figure & $\bar{\nu}_{0}\left(\mathrm{~cm}^{-1}\right)$ & $\Gamma$ & $\mathrm{C}$ & $\theta(\mathrm{rad})$ & $a$ \\
\hline 2d & 1181.33 & 111.81 & 0.42 & 0.54 & 1 \\
5d & 1729.98 & 27.21 & 3.06 & 1.46 & 1 \\
6a & 1726.23 & 20.91 & 0.50 & 0.43 & 1 \\
7b & 1257.16 & 16.53 & 1.44 & 0.39 & 1 \\
8a & 1313.81 & 6.41 & 0.49 & -0.91 & 2.95 \\
\hline \multirow{2}{*}{ 8c } & 1270.94 & 4.16 & 1.08 & 0.25 & 1.4 \\
& 1284.99 & 2.52 & 1.08 & 0.25 & 0.72 \\
\hline \multirow{2}{*}{ 8e } & 1523.30 & 30.40 & 3.39 & 0.9 & 0.53 \\
& 1612.75 & -7.99 & 3.39 & 0.9 & -0.23 \\
& 1636.10 & 41.5 & 3.39 & 0.9 & 0.99 \\
\hline
\end{tabular}

\section{Sample preparation}

We studied poly(methyl methacrylate) (PMMA) from $\mathrm{OCG}^{\mathrm{TM}}\left(\mathrm{OCG}^{\mathrm{TM}}\right.$ PMMA Arch 950K $9 \%$ resist, Chlorobenzene solution). PMMA/Au sample was prepared by spin-coating a 10 \% diluted PMMA-Chloroform solution at $9800 \mathrm{rpm}$ on Au. In the PDMS case, we firstly added $1 \%$ in mass of the Sylgard 184 curing agent to a PDMS solution, which was used to prepare a $5 \%$ diluted PDMS-toluene solution. In sequence, the sipn-coated PDMS/Au was made at $9000 \mathrm{rpm}$. Bovine Serum Albumin (BSA) from Sigma-Aldrich ${ }^{\circledR}$ (Fraction V $\geq 96$ \%) was diluted in Mili-Q water at the $4 \mathrm{mg} / \mathrm{ml}$ concentration. The BSA/Au sample was produced by drop-casting BSA solution onto Au substrate. 


\section{References}

(1) Hillenbrand, R.; Knoll, B.; Keilmann, F. Journal of Microscopy 2001, 202, 77-83.

(2) Hillenbrand, R.; Keilmann, F. Physical Review Letters 2000, 1-4.

(3) Keilmann, F.; Hillenbrand, R. Philosophical Transactions of the Royal Society of London A: Mathematical, Physical and Engineering Sciences 2004, 362, 787-805.

(4) Cvitkovic, A.; Ocelic, N.; Hillenbrand, R. Nano Letters 2007, 7, 3177-3181.

(5) Pollard, B.; Muller, E. A.; Hinrichs, K.; Raschke, M. B. Nature Communications 2014, 5,3587 . 